\title{
Design and Optimazation of the Shell for One Camera
}

\author{
Bo Gao ${ }^{1, a}$, HongtaoYang ${ }^{1,2}$, Kui Shi $^{1, b}$, Weining Chen ${ }^{1}$ \\ ${ }^{1}$ XI'AN Institute of optics andPrecision Mechanics of CAS, Xi'an, 710119, P.R.China. \\ ${ }^{2}$ Graduate School of Chinese Academy of Science, Beijing, 100864,P.R.China.



Keywords:Finite element analysis,structure optimization,the modal analysis.

\begin{abstract}
Thestructure of the shell designed for a camerawas investigated.The duralumin named 2A12-T4 was chosen as the main material in order to achievethe minimum weight,and the finite element optimization method wasused for the design of the shell.Introduced the structure characteristics of the shell.Discussed the using of optimization modulenamed Design Exploration in ANSYS workbench.Chosed the thickness of the shell wall and installationpointsas the main optimization parameters. We can see from the perspective of the result of optimization,the weight of shell was reduced from $320 \mathrm{~g}$ to $255 \mathrm{~g}$ than the original,reduced about $20 \%$ than before.In the same response spectrum,the stress and strain of the optimized structure are affordable and the result is better than before.It can meet the practical requirements.
\end{abstract}

\section{Introduction}

A lot of parts including Optical components and electronics components and so on are installed in the shell, So the camera shell is a major part of camera system. If thestiffness and strength of camera shell can not meet the actual demand,then it must be had a bad influencein imaging system and the imaging quality.So we make the wall thicker, the strength and stiffness can meet the requirements, but the weight will increase again.Because of this contradiction, we need to find a suitable means which not only can make the shell lightness,but also can meet the requirements of strength and stiffness. This paper is about the question. We use the optimization module of ANSYS workbench named Design Exploration to make the shell lighter, and the optimization results can meet the requirements of practical work, the shell has been put into production.

\section{The Design of the Camera Shell}

\subsection{The Choice of Material}

First of all,we should chose the material for the shell.We finally chosed the duraluminnamed 2A12-T4 as the main material for camera shellthrough the comparison of various materials performance. This kind of material is the main material of space products and it has many times flying experience. Its outstanding characteristicsare: mature technology, stable quality, relatively high stiffness and its density and the expansion coefficient are small, the main parameters of the material are shown in table $1^{[1]}$.

Table1 The main parameters of the 2A12-T4

\begin{tabular}{cccccc}
\hline name & $\begin{array}{c}\text { Desity } \\
(\mathrm{g} / \mathrm{cm} 3)\end{array}$ & $\begin{array}{c}\text { Poisson's } \\
\text { ratio }\end{array}$ & $\begin{array}{c}\text { Yield stress } \\
(\mathbf{M P a})\end{array}$ & $\begin{array}{c}\text { Expansion } \\
\text { coefficient }(\mathbf{K})\end{array}$ & $\begin{array}{c}\text { Modulus of } \\
\text { elasticity(GPa) }\end{array}$ \\
\hline 2A12-T4 & 2.77 & 0.3 & 325 & 22.7 & 71 \\
\hline
\end{tabular}

\subsection{The Design of Camera Shell}

The camera structure is mainly composed of shell, Cover plate, optical components, electronics components.It ishard to change the design of the optical components and electronics components to achieve the goal of weight loss.So we can only optimize the shell to achieve the goal.The camera structure shows in figure 1 , we are asked to make the weight of the camera less than $800 \mathrm{~g}$. The weight of electronics components, optical components, and other accessories is500g, so the weight 
of the shell must be less than 300g.In the design of the previous, we make the wall of shell thickness $3 \mathrm{~mm}$, the thickness of Installation points is $4 \mathrm{~mm}$. Camera is installed on the other equipment by the four installation points.

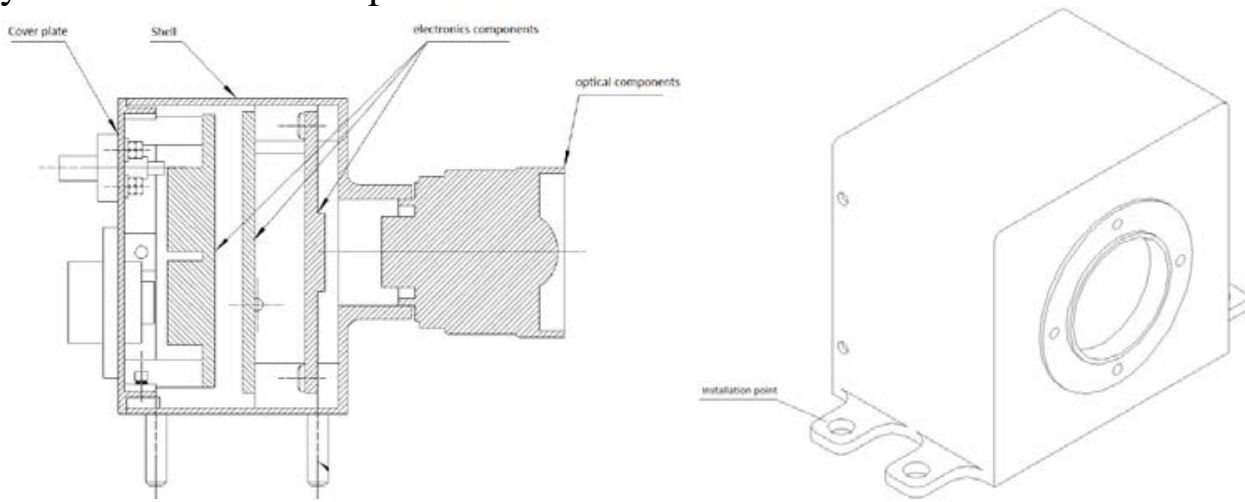

Figure 1The structure of camera

\subsection{The Finite Element Analysis Results of the Previously Camera Shell}

We make the dynamic response calculation for the shell to detect the performance of camera shell. Why must? Because its dynamic response is often could directly affect the dynamic performance of the camera.Therefore, the dynamic response analysis is particularly important in the designing of the whole camera.Modal analysis can fundamentally show the dynamic performance of the shell. The response spectrum analysis based on the result of modal analysis also can show the resistance performance of the shell. So in the paper,first of all, we make the modal analysis for the previous shell, and then make the response spectrum analysis.In the analysis results, the weight of the shell, resonance frequency, stress and strain areThe parameters of the main consideration.The camera shell must have the biggest frequency of first order when the least weight,but not only these,it also must have the suitable stress and strain. The weight of previous shell is $300 \mathrm{~g}$,the first order frequency is $1571 \mathrm{~Hz}$, the biggest shell deformation is $0.0045 \mathrm{~mm}$, the largest maximum stress is $8 \mathrm{Mpa}$, the results of modal analysis show in table 2.

Table2Theresults of first six order modal analysis

\begin{tabular}{ccccccc}
\hline order & $\mathbf{1}$ & $\mathbf{2}$ & 3 & 4 & 5 & $\mathbf{6}$ \\
\hline Frequency $(\mathrm{Hz})$ & 1571 & 1857 & 2888 & 3102 & 3420 & 3760 \\
\hline
\end{tabular}

We can see from the above analysis results,the first order frequency, stress and maximum deformation of the shell are allin the range of requirements, but the weight is slightly larger than the requirements, so we need to optimize the camera shell to reduce the weight.

\section{The Optimization Design of Camera Shell}

\subsection{Mathematical Description of the Structure Optimization}

The general mathematical expression for structure optimization design ${ }^{[2]}$ :

$$
\begin{gathered}
\text { design vector: } X=\left(x_{1}, x_{2} \cdots x_{n}\right)^{\top} \text {, } \\
\text { state vector: } Y=\left(y_{1}, y_{2} \cdots y_{n}\right)^{\top} \text {, }
\end{gathered}
$$

Minimize the objective function:

$$
\text { state equation: } \mathrm{f}_{\mathrm{k}}(\mathrm{X}, \mathrm{Y})=0(\mathrm{k}=1,2 \cdots \mathrm{m})
$$

constraint equation: $g_{j}(X, Y) \leqslant 0(j=1,2 \cdots n)$

There are three main elements in the mathematical description of the structure optimization design,they are the design variables objective function and constraint conditions. Ina structure optimization design scheme, according to the specific circumstances, the design variables can be the section size of the structure, area, the geometric parameters of the section ${ }^{[3]}$, moment of inertia and other parameters also can be such as elastic modulus of the material. The objective function is the main object of our optimization.it is one of the most importantCharacteristicsin the design of features, most of the structure design makethe lightest asthe objectives.Constraint condition is divided into boundary constraint and performance constraint. Boundary constraints are commonly used to limit the area of the change of the design variables and the relationship of the rules between 
a set of variables, the general presented show restraint in the form of performance constraint is according to design or performance indicators of a kind of constraint conditions, such as work stress, displacement, vibration frequency and buckling strength, etc., also known as the state variables. It is the dependent variable and it is a function of the design variables.So optimization problem in essence belongs to the category of mathematical programming, and complex structure optimization problem belongs to nonlinear programming.

\subsection{The optimization process of ANSYS workbench/Design Exploration}

We choose the lightest weight as the main objective function.The output variable P1 is the stress of the shell, the unit is the Mpa.The output variables P2 is the strain of the shell, the unit is mm.The input variables $\mathrm{d} 1$ is the thickness of the shell, input variable $\mathrm{d} 2$ is the thickness of the installation points, the constraints of optimization are the first-order natural frequency and the maximum stress, the maximum deformation.

The design mathematical description is as follows ${ }^{[4]}$ :

The objective function:Min $\Phi(\mathrm{X}, \mathrm{Y})$

the design variables: $\mathrm{X}=(\mathrm{d} 1, \mathrm{~d} 2)^{\mathrm{T}}$

The constraint: $0.5 \leqslant \mathrm{~d} 1 \leqslant 3.3$;

$$
\begin{gathered}
0.5 \leqslant \mathrm{~d} 2 \leqslant 4.4 ; \\
1400 \leqslant \mathrm{f} ; \\
\mathrm{P} 1 \leqslant 80 ;
\end{gathered}
$$

$\mathrm{P} 2 \leqslant 0.1 . \mathrm{f}$ is the first-order natural frequency.

\subsection{The results of finite element optimization}

We get the objective function and the first order frequency when constraint condition $\mathrm{d} 1, \mathrm{~d} 2$ changes, the result is shown in figure 2 .We can see from the analysis results when the shell thickness named d1turns to1.5 $\mathrm{mm}$, and the installation points named $\mathrm{d} 2$ turns to $2.5 \mathrm{~mm}$, the weight of the shell is $255 \mathrm{~g}$, the first order frequency is greater than $1400 \mathrm{~Hz}$, all above meet the requirements.



Figure2The objective function and the first order frequency

We make the response spectrum analysis to camera shell, the response spectrum is showed in figure 3 , the simulation results are shown in figure 4 .

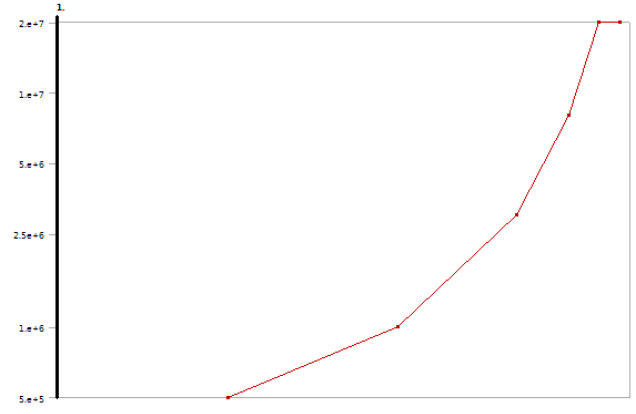

Figure3The response spectrum 


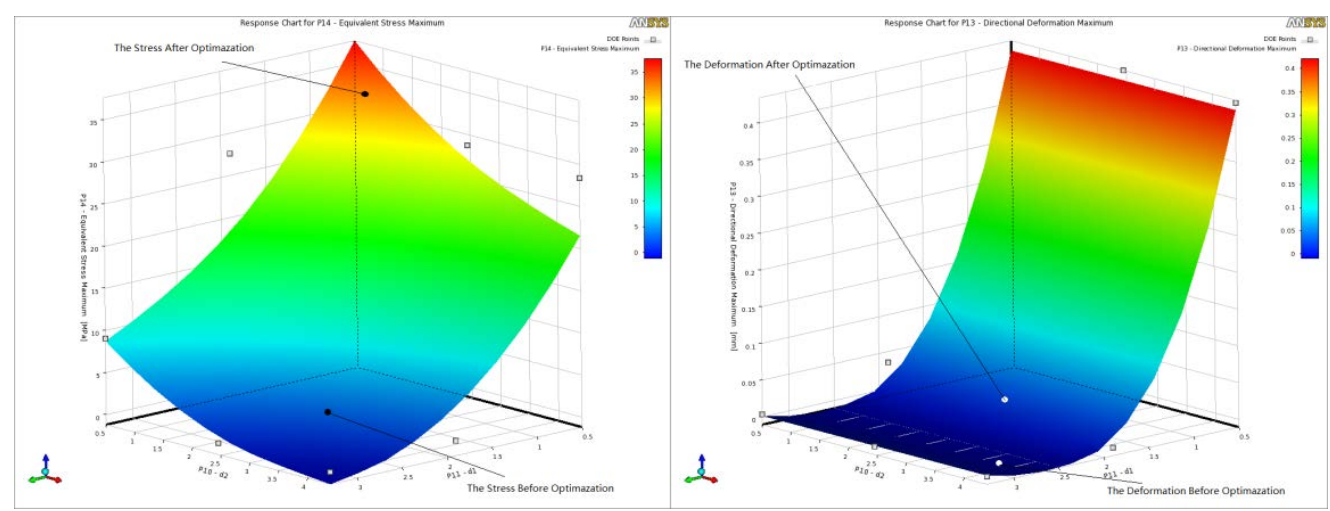

Figure4 Stress and deformation analysis of response spectrum

We can seethe simulation results of response spectrum from the figure above.We design the shell wall thickness $1.5 \mathrm{~mm}$ and the installation points $2.5 \mathrm{~mm}$, in this case, the maximum stress is only $112 \mathrm{Mpa}$, it is far less than the allowable stress of 2A12-T4, The maximum deformation of the shell is $0.014 \mathrm{~mm}$, less than the required $0.1 \mathrm{~mm}$.

\section{Conclusion}

In the traditional design, we want to reduce the time of designing work, the thickness of the shell wall and theInstallation points are often based on the design experience,so it will make materials waste.In this paper we use the finite element method to greatly reducethe weight of the camera shell under the condition of ensuring the stiffness and strength.We chose the duraluminnamed 2A12-T4 as the main material to meet thedesign requirements.We make the shell wall $1.5 \mathrm{~mm}$ and the installation points $2.5 \mathrm{~mm}$, in this case,the first order frequency of the shell is greater than $1400 \mathrm{~Hz}$, the weight of the design is only 255 g, relatively reduced $20.3 \%$ than the previous one.The camera shell which had been optimized have been put intoproduction ${ }^{[5]}$.

\section{References}

[1] XuezhiJia, Design and optimization oflightweight outer baffle for space camera[J].Optics and Precision Engineering,16(8),1560-1565， 2008.8

[2] WeizhiWang,WeijunGao,The Topology Optimization and Size Optimization in the Design of Space Camera Structure. Spacecraft Recovery \& Remote Sensing,33(6),67-73,2012.2

[3] Guangyipu,The Basic Tutorials and Example Explanation of ANSYS Workbench(M),China waterpower press,2013.4

[4] Dashan Dong, YuanyuanTeng, and Xiao Mei. The Application of ANSYS on the Teaching ofStructural Mechanics, Springer-Verlag Berlin Heidelberg 2011.

[5] ChangfengSun, The Structure Optimization Analysis of Optical Windows. Laser \& Infrared45(3),307-312,2015.3 